\title{
Comparison of the resistance of monolithic and layered heterogeneous barriers to penetration by rigid and deformable strikers with an ogival nose based on numerical simulation
}

\author{
Evgeny Kraus ${ }^{1}{ }^{*}$, and Ivan Shabalin ${ }^{1}$ \\ ${ }^{1}$ Khristianovich Institute of Theoretical and Applied Mechanics SB RAS, 630090, Novosibirsk, \\ Russia
}

\begin{abstract}
To create protective elements, information is needed on the resistance of various combinations of metal, ceramic, and heterogeneous plates, including layered ones, both in contact and with spacing. In addition, an intensive process is underway to create complex materials such as cermet composites. In this work, for verification of the code, numerical calculations of the breakdown of both monolithic and layered steel barriers by a striker with an ogival nose are performed. A series of 2D and 3D calculations for modeling the processes of penetrating heterogeneous barriers has been performed. It is shown that the resistance of heterogeneous barriers to penetration by a rigid striker is approximately the same, whereas the impact of a deformable striker on heterogeneous barriers shows that the resistance of layered barriers is lower compared to a monolithic barrier, and spacing of layers reduces the resistance of the barrier.
\end{abstract}

\section{Introduction}

The use of steel plates has found wide application in protective elements for any purpose, therefore, it has an extensive bibliographic history. Here are just a few classic works from this area. Thus, in [1,2], the resistance of Weldox 700E steel barriers to the action of a solid impactor with an ogival nose in the range of speeds not exceeding $450 \mathrm{~m} / \mathrm{s}$ was experimentally investigated. The following barriers were considered: (1L) a monolithic steel plate $12 \mathrm{~mm}$ thick, (2L) a two-layer barrier consisting of steel plates $2 \times 6 \mathrm{~mm}$ thick in contact, (1R) two-layer barrier consisting of steel plates $2 \times 6 \mathrm{~mm}$ with spacing of $12 \mathrm{~mm}$, and (2R) same barrier, but the layer spacing was $24 \mathrm{~mm}$. The perforation processes were calculated using LS-DYNA package. In general, good agreement was obtained between the experimental data and the results of numerical simulations. The finite elements modeling using appropriate material models allows one to capture the basic physical behavior during perforation of both monolithic and two-layer steel plates. All results and conclusions made in this study are based on a limited number of ballistic tests. The constructed ballistic

* Corresponding author: kraus@itam.nsc.ru 
curves for the studied barriers show that a monolithic steel plate is more resistant to twolayer barriers, both with contact of layers and with different spacing, whereas two-layer barriers have almost the same resistance. In [3], the ballistic resistance of one-, two-, three-, and four-layer steel plates to the action of strikers with lively warheads was experimentally investigated. Multilayer barriers with the arrangement of thicknesses in various combinations with the same total thickness were considered. Ballistic velocities were obtained for each obstacle configuration, the influence of air gaps between the layers, the number, order, and thickness of the layers on the ballistic resistance of each obstacle was determined. The conclusions of the work practically coincide with the results of $[1,2]$, namely, the monolithic targets have higher ballistic ultimate velocities than multilayer targets, the ballistic ultimate velocities of multilayer targets in contact are greater than those of spaced multilayer targets. The order of the layers affects the ballistic ultimate speeds of multilayer targets, the ballistic resistance of multilayer targets is better when the first layer is thinner than the second layer.

To reduce the specific weight of the protective elements, heterogeneous and gradient materials based on various metals and ceramics are being developed. Thus, in [4,5], a method was proposed for creating fundamentally new functionally gradient heterogeneous materials based on $\mathrm{B}_{4} \mathrm{C}$ ceramic powders with various mass fractions in the initial mixture and $\mathrm{Ni}$ plastic metal additive using the combined method of cold gas-dynamic spraying followed by layer-by-layer laser processing. It was shown that the microhardness of the track increases with increasing $\mathrm{B}_{4} \mathrm{C}$ concentration in the initial mixture. It was established that the structure of tracks depends on the size of ceramic particles in the range from 3 to 75 $\mu \mathrm{m}$. For the first time, a decrease in the size of $\mathrm{B}_{4} \mathrm{C}$ particles (by about 2-3 times) inside the track due to fragmentation under the action of a laser beam was observed.

Impact interaction of $\mathrm{SiC}$ spherical clusters with a rigid wall was studied in papers [6,7] by the molecular dynamics method. In [8], an experimental and theoretical analysis of the processes of penetration of a long rod into a multilayer barrier made of a metal-matrix composite (MMC) was carried out. MMC are potential materials for protective elements due to their light weight. Plates of two types of $\mathrm{MMC}$ made by liquid pressing: A356/45\%vol.SiC and A17075/45\%vol. $\mathrm{B}_{4} \mathrm{C}$ with a uniform distribution of $\mathrm{SiC}$ and $\mathrm{B}_{4} \mathrm{C}$ particles were studied. The mechanical properties were determined using a Hopkinson rod, hardness and compression tests, and the popular Johnson-Cook model was used for calculations. The front ceramic plate provides a slight increase in the effectiveness of the barrier by weight. A thin metal plate on top of the ceramic layer enhances the efficiency. Replacing the metal front layer with MMC shows the best mass efficiency without loss in the DoP test.

Several approaches have been proposed for modeling of MMC behavior in the process of deformation. The authors of [9] state that at the macro level, metal matrix composites (MMC) resemble homogeneous material, but at the micro level they have an inhomogeneous microstructure. The first step in the model development process is to decide on the size of the representative volume element (RVE). The further stages of the construction are clear from Fig. 3 from [9], where the geometric parameters for RVE are obtained from microphotographs using image processing software with open source software (OOF2) and MATLAB. Periodic boundary conditions have been successfully implemented in ABAQUS. The optimal parameter values for the Johnson-Cook model are found. The MMC model was satisfactorily implemented in plugin Materiales for ABAQUS.

In contrast to [9], the authors of [10-12] used a different approach: inclusions of another material, for example ceramics, were introduced directly into the metal matrix in the MMC numerical model. The model from [11,12] allows one to create directly heterogeneous and gradient materials according to a given law of the distribution of ceramic concentrations in the metal. According to approach [10], the volume of the composite at the micro level is 
modeled by a three-dimensional continuum, which is an aluminum matrix with embedded silicon carbide particles that occupy several finite elements. The microstructural properties of the metal matrix composite were selected in accordance with metallographic studies [13]. Software has been developed capable of generating three-dimensional models for structurally heterogeneous materials with a complex internal structure compatible with the input format of ANSYS package.

\section{Numerical simulation}

Based on the models and statement of the impact problems [14-18], 2D and 3D modeling of the processes of steel barriers perforation with a solid striker with an ogival nose was performed, according to the experimental data presented in [1,2]. The dimensions of the striker and barrier plates, as well as their mechanical properties are taken there. Hardened steel: yield strength is $1900 \mathrm{MPa}$, no information about fracture parameters; mild steel: yield strength is $850 \mathrm{MPa}$, fracture stress is $1550 \mathrm{MPa}$. The geometry of the problem for the $2 \mathrm{R}$ configuration in the $3 \mathrm{D}$ case is shown in Fig. 1.

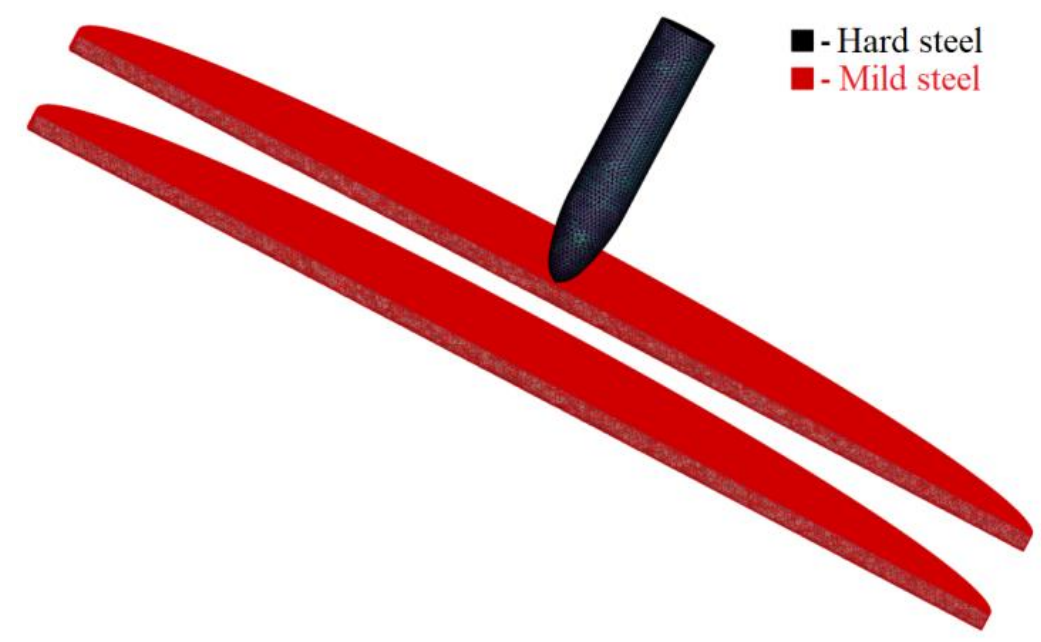

Fig. 1. The geometry of the problem of perforation of an obstacle from two steel plates spaced $24 \mathrm{~mm}$ apart.

The results of calculations of the $2 \mathrm{~L}$ configuration (a two-layer barrier with the contact of the layers), are shown in Figs. 2 and 3 in the 2D case, and in Fig. 4 for the 3D case, the impact velocity is $400 \mathrm{~m} / \mathrm{s}$. 


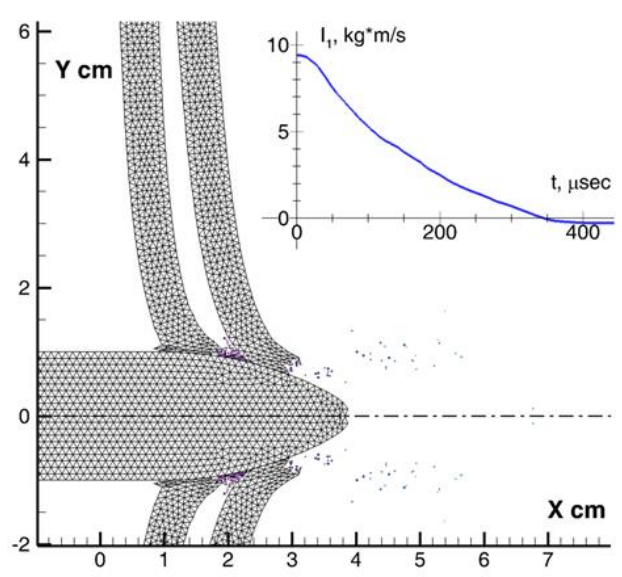

Fig. 2. The position of the hard striker in twolayer steel barrier with the contact of the layers at time $\mathrm{t}=400 \mu \mathrm{s}$ at the impact speed of $300 \mathrm{~m} / \mathrm{s}$ (cross-section along the axis with the difference grid), the graph in the right corner is the behavior of the momentum in time.

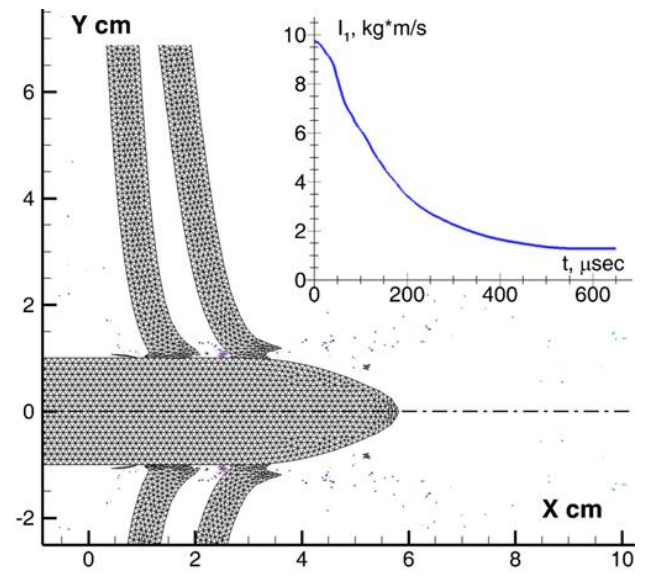

Fig. 3. The position of the hard striker in twolayer steel barrier with the contact of the layers at time $\mathrm{t}=500 \mu \mathrm{s}$ at the impact velocity of $310 \mathrm{~m} / \mathrm{s}$ (cross section along the axis with the difference grid), the graph in the right corner is the behavior of the momentum in time.
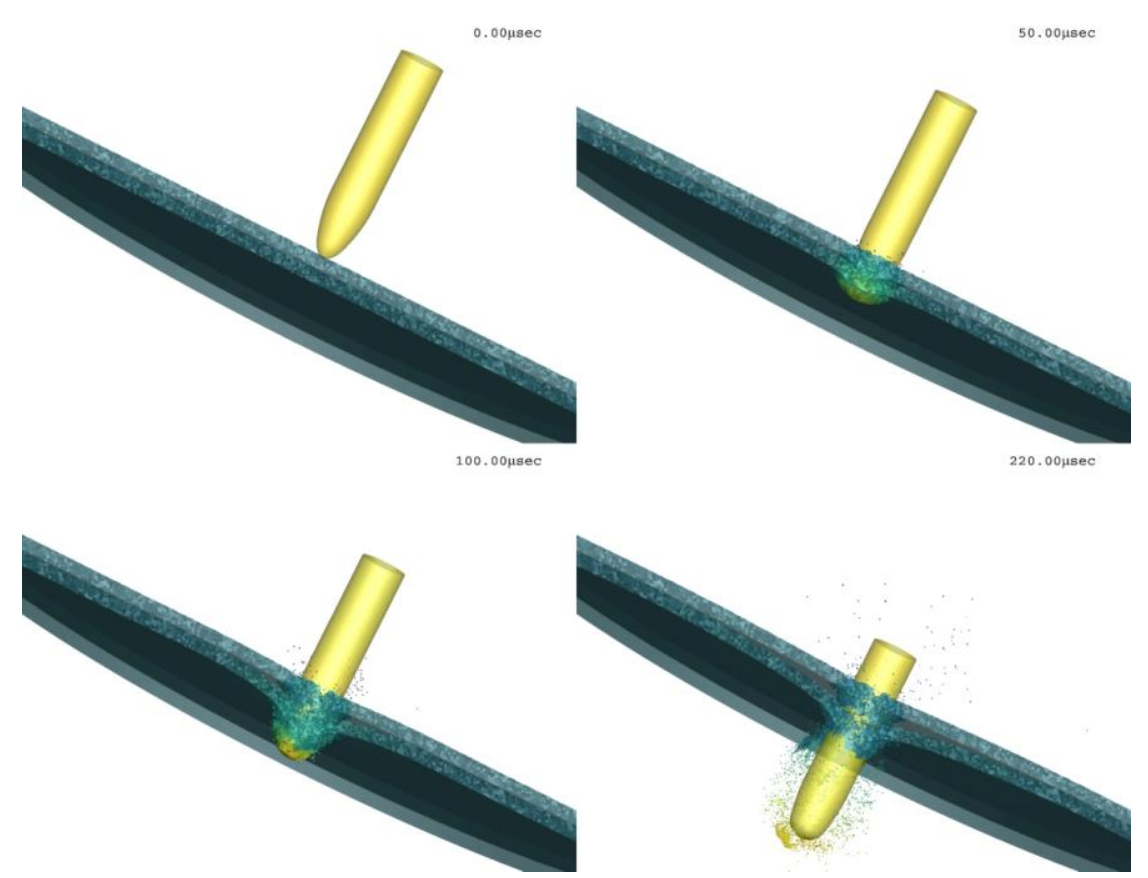

Fig. 4. The process of perforation of two-layer steel barrier with contact of the layers.

In the process of calculations, it turned out that the Prandtl - Reuss model with a constant yield strength does not ensure that the calculation results for the $2 \mathrm{R}$ configuration are consistent with the experimental data [1], while for a monolithic obstacle (1L configuration) it gives acceptable results. Based on a series of calculations with a variable yield strength, it was found out that to satisfy the experimental conditions, it is necessary to 
have $\mathrm{Y}=2.25 \mathrm{GPa}$ for the collision velocity $\mathrm{u}_{\mathrm{p}}=280 \mathrm{~m} / \mathrm{s}, \mathrm{Y}=2.05 \mathrm{GPa}$ for velocity $\mathrm{u}_{\mathrm{p}}=350 \mathrm{~m} / \mathrm{s}$, and $\mathrm{Y}=1.55 \mathrm{GPa}$ for velocity $\mathrm{u}_{\mathrm{p}}=450 \mathrm{~m} / \mathrm{s}$. The ballistic curves and calculation points are shown in Fig. 5 , where $u_{p}-$ projectile velocity, $u_{r}-$ residual velocity.

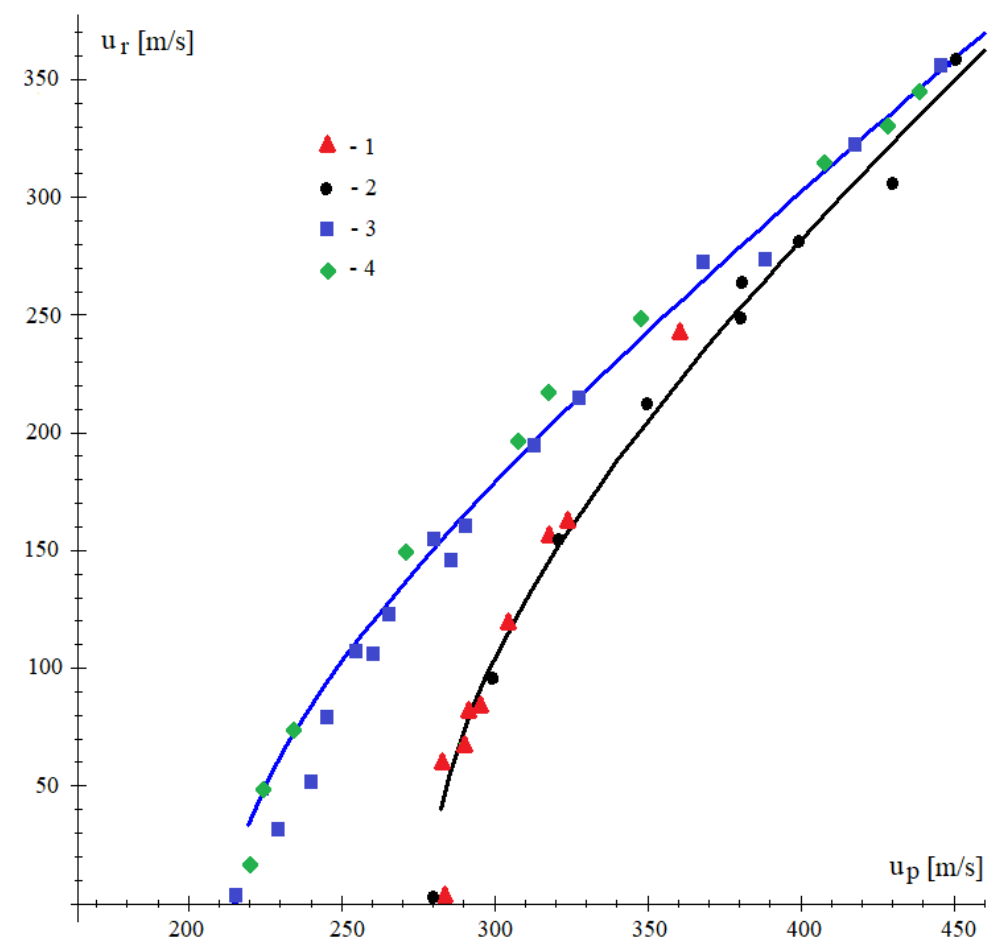

Fig. 5. Ballistic curves for the $2 \mathrm{R}$ configuration: two-layer steel barrier $2 \times 6 \mathrm{~mm}$ with $24 \mathrm{~mm}$ spacing. Designations: 1 - experimental data from [1], 2 - results of 2D calculations with variable yield strength $\mathrm{Y}, 3$ - results of $2 \mathrm{D}$ calculations with constant yield strength $\mathrm{Y}=860 \mathrm{MPa}, 4-3 \mathrm{D}$ calculation results, the curves are Lambert-Jonas functions approximating the data.

Heterogeneous barriers were built on the basis of the model from [11,19] with the conservation of weight, i.e. the barrier thickness was increased from $12 \mathrm{~mm}$ to $18.18 \mathrm{~mm}$, $2 \times 9.09 \mathrm{~mm}$ for two-layer barriers. Calculations were performed to compare the resistance of heterogeneous barriers similarly to the combinations of steel plates considered above, namely, a monolithic steel plate with the addition of $50 \%$ ceramic by volume (1L), the same material, but the barrier of two plates in contact (2L), two-layer barriers with plates spacing $12 \mathrm{~mm}(1 \mathrm{R})$ and $24 \mathrm{~mm}(2 \mathrm{R})$. The calculations were carried out both in 2D and 3D versions. Figures 6 and 7 show the geometry of 2D and 3D problems for heterogeneous barriers with $24 \mathrm{~mm}$ spacing. 


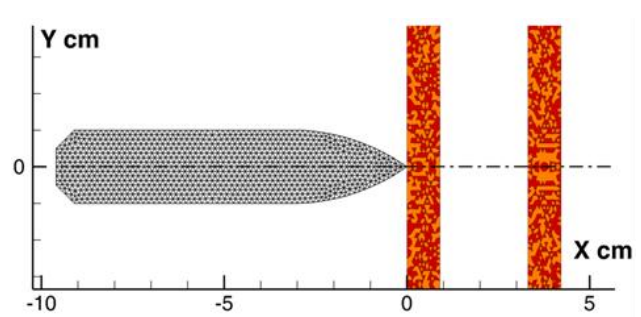

Fig. 6. The geometry of the 2D problem of perforation of a barrier from two spaced heterogeneous plates at distance of $24 \mathrm{~mm}$. Projectile - hard steel, plates - mixture mild steel with ceramic $\mathrm{B}_{4} \mathrm{C} 50 \%$ volume concentration.

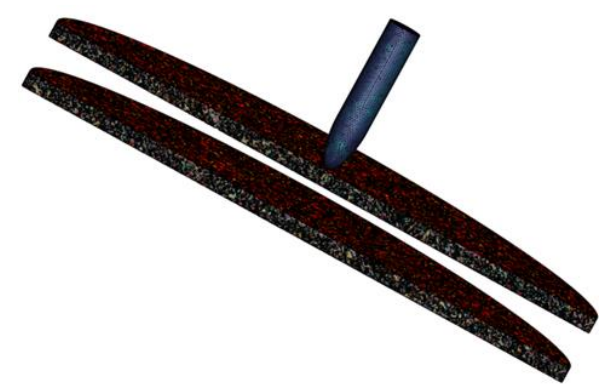

Fig. 7. The geometry of the 3D problem of perforation of a barrier from two spaced heterogeneous plates at distance of $24 \mathrm{~mm}$. Projectile - hard steel, plates - mixture mild steel with ceramic $\mathrm{B}_{4} \mathrm{C} 50 \%$ volume concentration.

The calculations were performed for two cases: in the first case, the yield strength of the impactor was overestimated to $\mathrm{Y}=5.0 \mathrm{GPa}$ (hard impactor), in the second case, $\mathrm{Y}=1.9 \mathrm{GPa}$ (deformable impactor). The results of model calculations revealed rather interesting trends in the resistance of heterogeneous barriers. The limiting ballistic velocity for both impactors is the same and is approximately equal to $\mathrm{ubl}=275 \mathrm{~m} / \mathrm{s}$. However, the behavior of the residual velocity is different: for the rigid impactor, all configurations of the barriers show practically the same resistance with an increase in the impact velocity (see Fig. 8). In the case of a deformable impactor, the ballistic curves with an increase in the impact velocity are a diverging set of curves, and the calculation results have a significant spread in the residual velocity (see Fig. 9). In Figures 8 and 9 are indicated: $1-$ monolithic obstacle, 2 - two-layer barrier with the layers contact, 3 - two-layer barrier with $24 \mathrm{~mm}$ spacing, 4 - two-layer barrier with $12 \mathrm{~mm}$ spacing. This is due to the random distribution of ceramic particles over the volume of the metal, because for the deformable impactor, the presence of ceramics in the front layers of the obstacle, especially the monolithic one, leads to deformation of the head part and an increase in the cross section, i.e. increase the resistance. Thin heterogeneous plates to a lesser extent deform the head of the projectile, and therefore the spread of the residual velocity is much lower. 


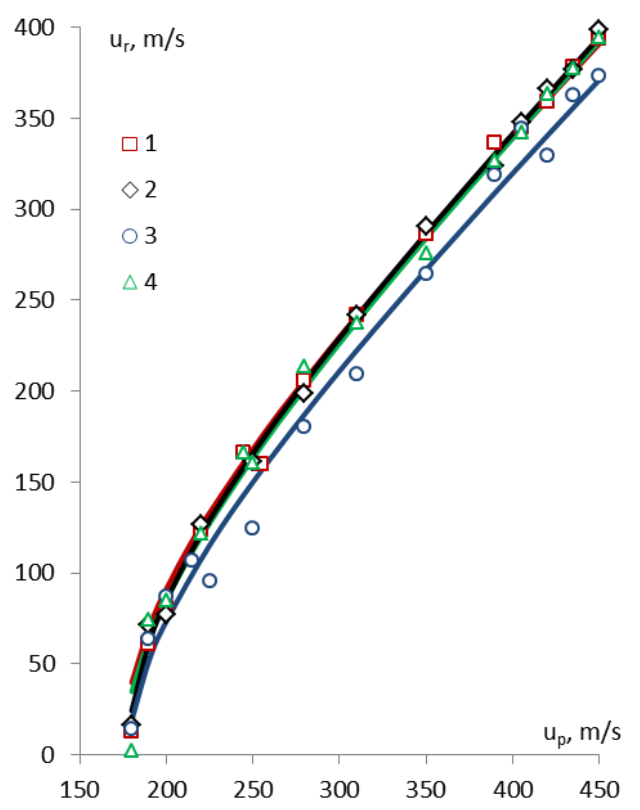

Fig. 8. Ballistic curves of perforation of heterogeneous barriers with the hard projectile.

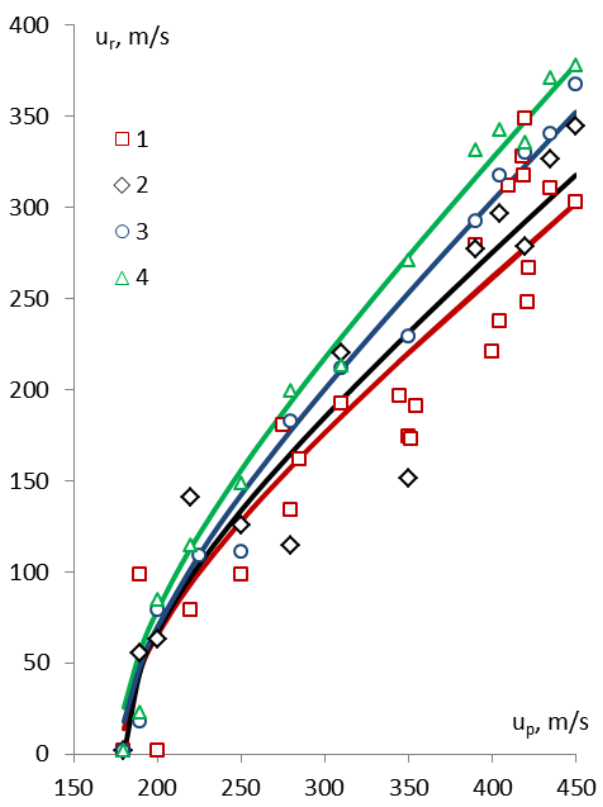

Fig. 9. Ballistic curves of perforation of heterogeneous obstacles by the deformable projectile.

\section{Conclusions}

Calculations with constant yield strength do not allow satisfactory agreement with the experimental data for spaced plates to be obtained.

Model calculations of the perforation of heterogeneous barriers based on metal-ceramic showed that for a rigid impactor, all configurations of barriers have the same resistance over a wide range of impact velocity. At that, as for a deformable impactor, the ballistic curves with an increase in the impact velocity are a diverging set of curves

The ballistic limit velocity for both impactors and all configurations of barriers is practically the same.

The work is supported by the SB RAS Interdisciplinary project "Development and synthesis of new functional gradient materials" (No. AAAA-A17-117030610136-3).

\section{References}

1. S. Dey, T. Børvik, and T. Borvik, "Ballistic Penetration and Perforation of Layered Steel Plates: An Experimental and Numerical Investigation," in 23rd International Symposium on Ballistics, F. Galvez, ed., INTERNATIONAL SYMPOSIUM ON BALLISTICS (Francisco Galvez, 2007), pp. 1365-1372

2. S. Dey, T. Børvik, X. Teng, T. Wierzbicki, and O. S. Hopperstad, Int. J. Solids Struct. 44(20), 6701-6723 (2007)

3. Y. Deng, W. Zhang, and Z. Cao, Mater. Des. 44, 228-239 (2013)

4. V. M. M. Fomin, A. A. A. Golyshev, V. F. F. Kosarev, A. G. G. Malikov, A. M. M. Orishich, N. S. S. Ryashin, A. A. A. Filippov, and V. S. S. Shikalov, J. Appl. Mech. 
Tech. Phys. 58(5), 947-955 (2017)

5. A. A. Filippov, V. M. Fomin, A. G. Malikov, and A. M. Orishich, Nanomechanics Sci. Technol. An Int. J. 8(1), 55-66 (2017)

6. A. V. Utkin and V. M. Fomin, AIP Conf. Proc. 1893, 030018 (2017)

7. A. V. Utkin, V. M. Fomin, and V. B. Utkin, AIP Conf. Proc. 2027, 030153 (2018)

8. M. Lee, S. Park, I. Jo, and S. Lee, in Procedia Engineering (Elsevier Ltd, 2017), 204, pp. 100-107

9. J. L. York Duran, C. Kuhn, and R. Müller, Appl. Mech. Mater. 869, 94-111 (2017)

10. S. V. Smirnov, A. V. Konovalov, M. V. Myasnikova, Y. V. Khalevitsky, and A. S. Smirnov, in IOP Conference Series: Materials Science and Engineering (Institute of Physics Publishing, 2017), 208(1)

11. E. I. Kraus, I. I. Shabalin, and T. I. Shabalin, AIP Conf. Proc. 1893, 030130 (2017)

12. E. I. Kraus and I. I. Shabalin, AIP Conf. Proc. 2125, 030065 (2019)

13. N. B. Pugacheva, N. S. Michurov, and T. M. Bykova, Phys. Met. Metallogr. 117(6), 634-640 (2016)

14. M. L. Wilkins, Computer Simulation of Dynamic Phenomena, Scientific Computation (Springer Berlin Heidelberg, 1999)

15. E. I. Kraus and I. I. Shabalin, Frat. ed Integrita Strutt. 24, (2013).

16. V. M. Fomin, A. I. Gulidov, G. A. Sapozhnikov, and I. I. Shabalin, High-Velocity Solids Interaction (SB RAS, 1999)

17. E. I. Kraus and I. I. Shabalin, Math. Montisnigri 39, 18-29 (2017)

18. A. V. Radchenko, P. A. Radchenko, and S. P. Batuev, Russ. Phys. J. 58(3), 319-329 (2015)

19. E. I. Kraus, A. Y. Melnikov, V. M. Fomin, and I. I. Shabalin, J. Appl. Mech. Tech. Phys. 60(3), 526-532 (2019) 